\title{
1. Rogue waves in shallow water in the presence of a 2 vertically sheared current
}

\author{
3 Christian Kharif • Malek Abid • Julien \\ 4 Touboul \\ 6 Received: date / Accepted: date
}

7 Abstract Two-dimensional rogue wave occurrence in shallow water on a ver8 tically sheared current of constant vorticity is considered. Using Euler equa-

9 tions and Riemann invariants in the shallow water approximation, hyperbolic 10 equations for the surface elevation and the horizontal velocity are derived and 11 closed-form nonlinear evolution equation for the surface elevation is obtained. 12 Following Whitham (1974), a dispersive term is added to this equation us${ }_{13}$ ing the fully linear dispersion relation. With this new single first-order partial

C. Kharif

Aix Marseille Université, CNRS, Centrale Marseille, IRPHE UMR 7342, F-13384, Marseille, France

E-mail: kharif@irphe.univ-mrs.fr

M. Abid

Aix Marseille Université, CNRS, Centrale Marseille, IRPHE UMR 7342, F-13384, Marseille, France

J. Touboul

Université de Toulon, Aix Marseille Université, CNRS, IRD, Mediterranean Institute of Oceanography (MIO), La Garde, France

Aix Marseille Université, Université de Toulon, CNRS, IRD, Mediterranean Institute of Oceanography (MIO), Marseille, France 
differential equation, vorticity effects on rogue wave properties are studied numerically. Besides, the Boundary Integral Element Method (BIEM) and the $\mathrm{KdV}$ equation both with vorticity are used for this numerical investigation, too. It is shown that results from the generalised Whitham equation agree quite well with those from BIEM whereas those from the KdV model are quite different. The numerical simulations carried out with the generalised Whitham equation and BIEM show that the presence of an underlying vertically sheared current modifies rogue wave properties significantly. For negative vorticity the amplification factor and duration of extreme wave events are increased whereas it is the opposite for positive vorticity.

\section{Introduction}

Generally, in coastal and ocean waters, current velocity profiles are established by bottom friction and wind stress at the sea surface, and consequently are vertically varying. Ebb and flood currents due to the tide may have an important effect on water wave properties. In any region where the wind blows, the generated current affects the behavior of the waves. The present work focuses on the nonlinear evolution of two-dimensional gravity waves propagating in shallow water on a shear current which varies linearly with depth. We assume that the directional spread of the wave field is sufficiently narrow to consider unidirectional propagation of the waves.

There are a number of physical mechanisms that focus the wave energy into a small area and produce the occurrence of extreme waves called freak or rogue waves. These events may be due to refraction (presence of variable currents or bottom topography), dispersion (frequency modulation), wave instability (the modulational instability), soliton interactions, crossing seas, etc. For more details on these different mechanisms see the reviews on freak waves by Kharif and Pelinovsky (2003), Dysthe et al (2008), Kharif et al (2009) and Onorato et al (2013). Few studies have been devoted to the occurrence of extreme wave events in shallow water. Among the authors who have investigated rogue wave 
43 properties in shallow water, one can cite Pelinovsky et al (2000), Kharif et al

44 (2000), Peterson et al (2003), Soomere and Engelbrecht (2005), Talipova et al

45 (2008) and Chambarel et al (2010). Pelinovsky and Sergeeva (2006) and Toffoli

46 et al (2006) investigated the statistical properties of rogue waves in shallow

47 water.

48 To the best of our knowledge, there is no paper on the effect of a vertically

49 sheared current on rogue wave properties apart from that of Touboul and

50 Kharif (2016) in deep water. We propose to extend this work to the case of

51 shallow water.

${ }_{52}$ Within the framework of the shallow water wave theory Whitham (1974) pro-

53 posed a generalised equation governing the evolution of fully nonlinear waves

54 satisfying the full linear dispersion. The Whitham equation may be derived

55 from the previous generalised Whitham equation assuming that the waves

56 are weakly nonlinear. The Whitham equation and the KdV equation which

57 have the same nonlinear term differ from each other by the dispersive term.

${ }_{58}$ Very recently, Hur and Johnson (2015) have considered a modified Whitham

59 equation taking account of constant vorticity. Very recently, Kharif and Abid

${ }_{60}$ (2017) have proposed a new model derived from the Euler equations for wa-

61 ter waves propagating on a vertically sheared current of constant vorticity in

62 shallow water. The heuristic introduction of dispersion allows the study of

${ }_{63}$ strongly nonlinear two-dimensional long gravity waves in the presence of vor-

64 ticity. Consequently, this new equation extends to waves propagating in the

65 presence of vorticity the generalised Whitham equation.

${ }_{66}$ Two different approaches are used to investigate rogue waves propagating

67 in shallow water on a shear current of constant vorticity: the generalised

${ }_{68}$ Whitham equation with vorticity and the Boundary Integral Element Method

69 (BIEM) which allows the study of fully nonlinear dispersive water waves on

70 arbitrary depth in the presence of vorticity (see Touboul and Kharif (2016)).

71 Besides, a numerical investigation is carried out by using the $\mathrm{KdV}$ equation

72 with constant vorticity whose derivation can be found in the papers by Free- 
73 man and Johnson (1970) and Choi (2003). Note that the latter equation can

74 be derived from the generalised Whitham equation with vorticity assuming

75 that the waves are weakly nonlinear and weakly dispersive.

\section{Two mathematical formulations}

$77 \quad 2.1$ The generalised Whitham equation with vorticity

83 The continuity equation is

$$
u_{x}+w_{z}=0
$$

84

$$
\eta_{t}+\frac{\partial}{\partial x}\left[u(\eta+h)+\frac{\Omega}{2} \eta^{2}+U_{0} \eta\right]=0
$$

89 where $u$ is assumed to be independent of $z$.

90 Equation (2) corresponds to mass conservation in shallow water in the pres91 ence of constant vorticity.

92 Under the assumption of hydrostatic pressure, the Euler equation in $x$-direction 93 is

$$
u_{t}+\left(u+U_{0}+\Omega z\right) u_{x}+\Omega w+g \eta_{x}=0
$$

94 where $g$ is the gravity. 
Using the continuity equation and boundary conditions that $w$ satisfies on the bottom and at the free surface, we obtain

$$
w=-(z+h) u_{x}
$$

It follows that the Euler equation becomes

$$
u_{t}+\left(u+U_{0}-\Omega h\right) u_{x}+g \eta_{x}=0
$$

The dynamics of non dispersive shallow water waves on a vertically sheared current of constant vorticity is governed by equations (2) and (5) that admit a pair of Riemann invariants. These Riemann invariants which are derived analytically allows us to express the longitudinal component of the wave induced velocity $u(x, t)$ as a function of the elevation $\eta$. Finally, equations (2) and (5) can be reduced to the following single nonlinear partial differential equation for $\eta$

$$
\begin{gathered}
\eta_{t}+\left\{U_{0}-\frac{\Omega h}{2}+2 \sqrt{g(\eta+h)+\Omega^{2}(\eta+h)^{2} / 4}-\sqrt{g h+\Omega^{2} h^{2} / 4}+\right. \\
\left.\frac{g}{\Omega} \ln \left[1+\frac{\Omega}{2 g} \frac{\Omega \eta+2\left(\sqrt{g(\eta+h)+\Omega^{2}(\eta+h)^{2} / 4}-\sqrt{g h+\Omega^{2} h^{2} / 4}\right)}{1+\frac{\Omega}{g}\left(\frac{\Omega h}{2}+\sqrt{g h+\Omega^{2} h^{2} / 4}\right)}\right]\right\} \eta_{x}=0
\end{gathered}
$$

This equation is fully nonlinear and describes the spatio-temporal evolution of hyperbolic water waves propagating rightwards in shallow water in the presence of constant vorticity.

Following Whitham (1974), full linear dispersion is introduced heuristically

$$
\begin{array}{r}
\eta_{t}+\left\{U_{0}-\frac{\Omega h}{2}+2 \sqrt{g(\eta+h)+\Omega^{2}(\eta+h)^{2} / 4}-\sqrt{g h+\Omega^{2} h^{2} / 4}\right. \\
\left.\frac{g}{\Omega} \ln \left[1+\frac{\Omega}{2 g} \frac{\Omega \eta+2\left(\sqrt{g(\eta+h)+\Omega^{2}(\eta+h)^{2} / 4}-\sqrt{g h+\Omega^{2} h^{2} / 4}\right)}{1+\frac{\Omega}{g}\left(\frac{\Omega h}{2}+\sqrt{g h+\Omega^{2} h^{2} / 4}\right)}\right]\right\} \eta_{x}+K * \eta_{x}=0
\end{array}
$$

where $K * \eta_{x}$ is a convolution product. The kernel $K$ is given as the inverse Fourier transform of the fully linear dispersion relation of gravity waves in 
finite depth in the presence of constant vorticity $\Omega: K=F^{-1}(c)$ with

$$
c=\sqrt{g h}\left(\sqrt{\frac{\tanh (k h)}{k h}\left(\frac{\Omega^{2} \tanh (k h)}{4 g k}+1\right)}-\frac{\Omega \tanh (k h)}{2 k \sqrt{g h}}\right)
$$

Equation (7) governs the propagation of nonlinear long gravity waves in a fully linear dispersive medium. For $\Omega=0$ and $U_{0}=0,(6)$ reduces to generalised equation (13.97) of Whitham (1974).

For weakly nonlinear $(\eta / h \ll 1)$ and weakly dispersive $(k h \ll 1)$ waves, equation (7) reduces to the KdV equation with vorticity derived by Freeman and Johnson (1970) and Choi (2003) who used multiple scale methods, different to the approach used herein. To set the KdV equation in dimensionless form, $h$ and $\sqrt{h / g}$ are chosen as reference length and reference time which corresponds to $h=1$ and $g=1$. The equation reads

$$
\eta_{t}+c_{0}(\Omega) \eta_{x}+c_{1}(\Omega) \eta \eta_{x}+c_{2}(\Omega) \eta_{x x x}=0
$$

with

$c_{0}=U_{0}-\frac{\Omega}{2}+\sqrt{1+\Omega^{2} / 4} \quad, \quad c_{1}=\frac{3+\Omega^{2}}{\sqrt{4+\Omega^{2}}} \quad, \quad c_{2}=\frac{2+\Omega^{2}-\Omega \sqrt{4+\Omega^{2}}}{6 \sqrt{4+\Omega^{2}}}$

The equations (6), (7) and (8) are solved numerically in a periodic domain of length $2 L$. The length $L$ is chosen $O(400 \delta)$ where $\delta$ is a characteristic length scale of the initial condition. The number of grid points is $N_{x}=2^{12}$. Spatial derivatives are computed in the Fourier space and nonlinear terms in the physical space. The link between the two spaces is made by the Fast Fourier Transform. For the time integration, a splitting technique is used. The equations (6), (7) and (8) could be written as

$$
\eta_{t}+L+N=0
$$

where $L$ and $N$ are linear and nonlinear differential operators in $\eta$, respectively.

Note that in general the operators $L$ and $N$ do not commute. If the initial condition is $\eta_{0}$, the exact solution of the previous equation is

$$
\eta(t)=e^{-(L+N) t} \eta_{0}
$$


This equation is discretized as follows. Let $t_{n}=n \Delta t$. We have

$$
\eta\left(t_{n}\right)=e^{-(L+N) n \Delta t} \eta_{0}=\left(e^{-L \Delta t / 2} e^{-N \Delta t} e^{-L \Delta t / 2}\right)^{n} \eta_{0}+O\left(\Delta t^{2}\right),
$$

and the scheme is globally second order in time. The operator $e^{-L \Delta t / 2}$ is computed exactly in the Fourier space. However, the operator $e^{-N \Delta t}$ is approximated using a Runge-Kutta scheme of order 4 . The time step is chosen as $\Delta t=0.005$. Furthermore, the efficiency and accuracy of the numerical method has been checked against the nonlinear analytical solution of the StVenant equations for the dam-break problem in the absence of current and vorticity ( $\Omega=0$ and $U_{0}=0$ ). For $U_{0}=0$ and $\Omega=0$ equation (6) reduces to

$$
H_{t}+(3 \sqrt{g H}-2 \sqrt{g h}) H_{x}=0, \quad \text { with } \quad H=\eta+h .
$$

For $t>0$, the nonlinear analytical solution of equation (12) is

$$
\begin{aligned}
H(x, t)=h, & u(x, t)=0 ; & \frac{x}{t} \geq \sqrt{g h} \\
H(x, t)=\frac{h}{9}\left(2+\frac{x}{\sqrt{g h} t}\right)^{2}, & u(x, t)=-\frac{2}{3}\left(\sqrt{g h}-\frac{x}{t}\right) & ;-2 \sqrt{g h} \leq \frac{x}{t} \leq \sqrt{g h} \\
H(x, t)=0, & u(x, t)=0 ; & \frac{x}{t} \leq-2 \sqrt{g h}
\end{aligned}
$$

At time $t=0$ the initial condition is $H(x, 0)=h(1+\tanh (2 x)) / 2$ and $u(x, 0)=$ 0 everywhere. A numerical simulation of equation (12) has been carried out with $g=1$ and $h=1$. The numerical and analytical surface profiles at $t=0$ and after the dam has broken are plotted in figure 1.

Within the framework of the KdV equation in the presence of vorticity, we have also checked that solitary waves are propagated with the right velocity that depends on $\Omega$.

\subsection{The boundary Integral Element Method}

The problem considered here is identical to the one described in the previous section. It is two dimensional, and the current field is assumed to be steady, constant in the horizontal direction, and to vary linearly with depth,

$$
U(z)=U_{0}+\Omega z .
$$




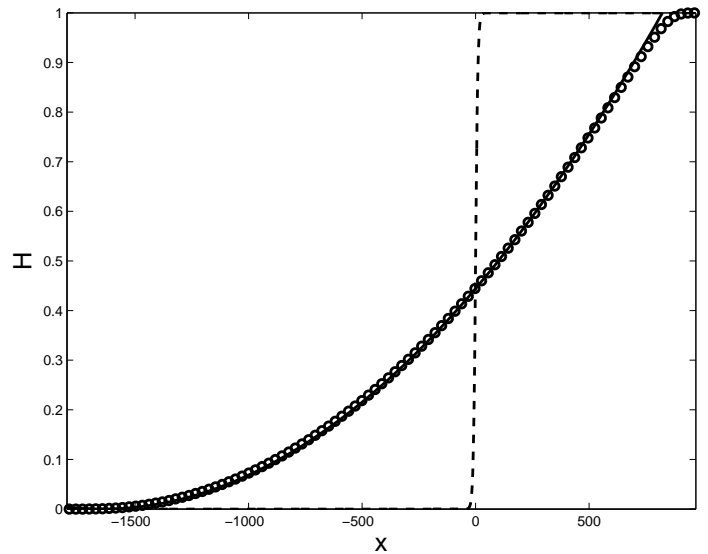

Fig. 1 Dam-break: comparison between analytical (solid line) and numerical solutions (o) after the dam has broken. The dashed line represents the initial condition at $t=0$

$$
\begin{aligned}
& \tilde{u}(x, z, t)=u(x, z, t)+U(z), \\
& \tilde{v}(x, z, t)=v(x, z, t) \quad \text { and } \\
& \tilde{p}(x, z, t)=p(x, z, t)+P(z) .
\end{aligned}
$$

Using this decomposition, the Euler equations might reduce to

$$
\begin{aligned}
u_{t}+(U+u) u_{x}+v U_{z}+v u_{z} & =-\frac{p_{x}}{\rho} \quad \text { and } \\
v_{t}+(U+u) v_{x}+v v_{z}+g & =-\frac{p_{z}}{\rho},
\end{aligned}
$$

which has to be fulfilled together with the continuity equation

$$
u_{x}+v_{z}=0
$$


As it is demonstrated in Simmen (1984), and more recently in Nwogu (2009), the wavy perturbations propagating in such current conditions are irrotational. Indeed, since the second derivative of the background current $U_{z z}$ is nil, the vorticity conservation equation involves no source term, and the vorticity field does not exchange any vorticity with the wavy perturbations. Thus, we might introduce a velocity potential $\phi(x, z, t)$ from which derive the perturbation induced velocities $(\nabla \phi=(u, v))$. It has to be emphasized that the continuity equation (18) is automatically satisfied if the velocity potential is solution of Laplace's equation

$$
\Delta \phi=0 .
$$

The kinematic free surface condition might also be expressed, and if $(X, Z)$ denotes the location of a particle at the free surface, this condition might be expressed

$$
\frac{d X}{d t}=u \quad \text { and } \quad \frac{d Z}{d t}=v-U(\eta) \frac{\partial \eta}{\partial x},
$$

where $d / d t$ refers to the material derivative $d / d t=\partial / \partial t+u \partial / \partial x+v \partial / \partial z$, and $Z=\eta(x, t)$.

Now, a stream function $\psi$ can also be introduced, so that $(\partial \psi / \partial z,-\partial \psi / \partial x)=$ $(u, v)$. The Euler equations (16) and (17) can now be integrated in space, and it comes

$$
\frac{\partial \phi}{\partial t}+U(z) \frac{\partial \phi}{\partial x}+\frac{\nabla \phi^{2}}{2}-\Omega \psi+g z=-\frac{p}{\rho}
$$

When applied to the free surface, where the pressure is constant, this equation provides the classical dynamic boundary condition. Introducing the material derivative used in the kinematic condition, this condition reduces to

$$
\frac{d \phi}{d t}+U(\eta) \frac{\partial \phi}{\partial x}-\frac{\nabla \phi^{2}}{2}-\Omega \psi+g \eta=0,
$$

At this point, the knowledge of the stream function $\psi$ at the free surface is still needed. Hopefully, one can notice the relationship

$$
\frac{\partial \psi}{\partial \tau}=-\frac{\partial \phi}{\partial n},
$$

where $(\boldsymbol{\tau}, \mathbf{n})$ refer respectively to the tangential and normal vectors at the free surface. Thus, the stream function $\psi$ can be evaluated at the free surface as 
soon as the normal derivative of the velocity potential is known.

Furthermore, if equations (20), (22) and (23) refer to the boundary condition at the free surface, the fluid domain still has to be closed. This is done by using impermeability conditions on the bottom boundary condition, located at $z=-h, h$ being used as the reference length (i.e. $h=1)$ and on the vertical boundary conditions, located respectively at $x=0$ and $x=200$.

The numerical approach used here has already been implemented and used successfully in the framework of focusing wave groups in the presence of uniform current (Touboul et al (2007); Merkoune et al (2013)). The extension allowing to take constant vorticity into account was presented in Touboul and Kharif (2016) together with a validation of the approach. It is based on a Boundary Integral Element Method (BIEM) coupled with a Mixed Euler Lagrange (MEL) procedure. At each time step, the Green's second identity is discretized to solve numerically the Laplace equation (19). Thus, the potential and its normal derivative are known numerically, and the stream function $\psi$ can be deduced by integration of equation (23) along the free surface. This numerical integration is performed in the up-wave direction, starting from the down-wave end of the basin, and using zero as initial value. Then, the time stepping is performed by numerical integration of equations (20) and (22) using a fourth order Runge \& Kutta scheme. Full details of the implementation can be found in Touboul and Kharif (2010). In every simulations, the total number of points considered at the free surface was $N_{f s}=1000$, while the total number of points used on the solid boundaries was $N_{b o}=600$. The time step used for the simulations was $d t=0.01$.

\subsection{Initial condition}

Both numerical approaches described in previous subsections were initialised with the same intial condition. Following the approach described in Kharif et al (2000); Pelinovsky et al (2000), the initial condition is obtained numerically.

A Gaussian initial wave, with no initial velocity, is allowed to collapse under 
gravity. This simulation is run in the absence of current and vorticity, using

the BIEM. Two radiated wave trains, propagating in opposite directions, are generated. The wave group propagating in the $(-x)$ direction is isolated, and space-time coordinates are reverted. This allows the generation of a focusing wave group in shallow water conditions. For the numerical simulations considered here, the initial gaussian elevation has a maximum amplitude $a=h$ where $h$ still being the reference length, and a width $\sigma=2 h$.

The wave train considered is used as initial condition for both numerical approaches. The surface elevation of this focusing wave group is used as initial condition for the generalised-Whitham equation with vorticity, and for the $\mathrm{KdV}$ equation with vorticity as well. Both elevations and velocity potential are required to initialise the BIEM.

The dimensionless value of the maximum surface elevation of the wave group obtained, $\eta_{\max }(t=0)$, is 0.0715 . The dynamics of this wave packet is illustrated in figure 2, in the framework of BIEM simulations. The initial wave packet is propagated, and the effects of both nonlinearity and dispersion lead to the formation of a high wave.

\section{Results and discussion}

Among the rogue wave properties, a particular attention is paid to the amplification factor of the maximum surface elevation, defined as $\eta_{\max }(t) / \eta_{\max }(t=$ 0 ). The time evolution of this amplification factor is plotted in figures 3-7 for several values of the shear $\Omega$. One can see that the evolutions computed with the generalised Whitham equation and BIEM are similar even though the amplification is overestimated with the generalised Whitham equation with vorticity. The amplification factor at the focusing time $t_{f}$ plotted in figure 8 increases as the shear $\Omega$ increases. One can observe that the difference between the two curves decreases as the shear $\Omega$ increases. In other words, the agreement is better for positive values of the shear $\Omega$ (negative vorticity) than for negative values of $\Omega$ (positive vorticity). The $\mathrm{KdV}$ equation exhibits the same 

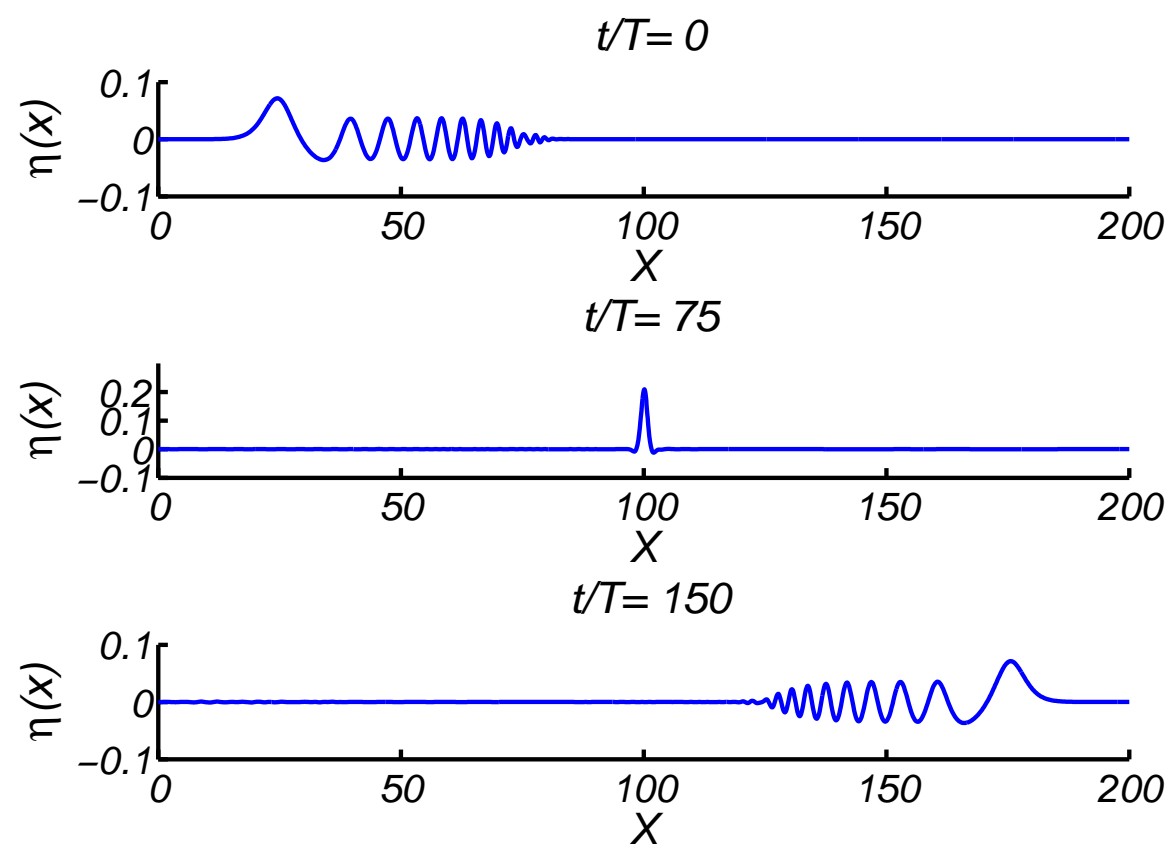

Fig. 2 Surface elevation of the focusing waves group evolving from initial condition $(t / T=$ $0)$ to rogue wave occurence $(t / T=75)$, before defocusing $(t / T=150)$.

tendency that is an increase of the maximum of amplification with $\Omega$. The focusing time $t_{f}$ obtained with both models are very close. On the opposite, the $\mathrm{KdV}$ equation underestimates the maximum value of the amplification factor and the focusing time $t_{f}$ as well. In figures 6 and 7 , the BIEM shows for negative values of the shear $\Omega$ first a reduction of the maximum surface elevation and then an amplification. This attenuation of the maximum of the surface elevation does not occur for the generalised Whitham and KdV equations. We define as extreme wave events or rogue waves those in the group whose surface elevation satisfies $\eta_{\max }(t=0) / \eta_{\max }(t) \geq 2$. In that way, we can introduce the rogue wave lifetime which is the duration of the extreme wave event. In figure 9 is shown this duration as a function of $\Omega$. For positive values of the shear $\Omega$ the rogue wave duration is increased whereas it is the opposite for negative values. 


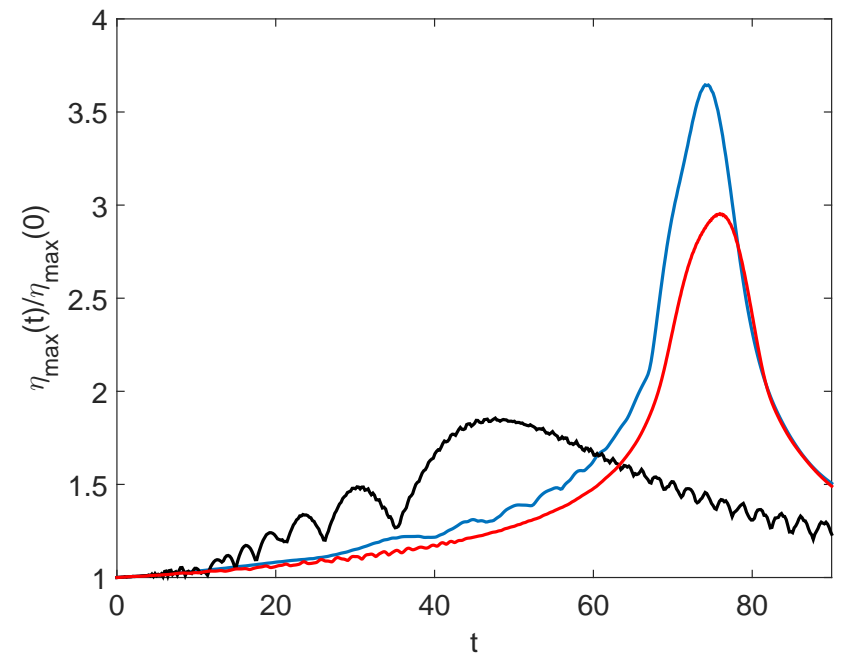

Fig. 3 (color online) Time evolution of the amplification factor without vorticity effect $(\Omega=0)$. Generalised Whitam equation (blue solid line), BIEM (red solid line) and KdV equation (black solid line)

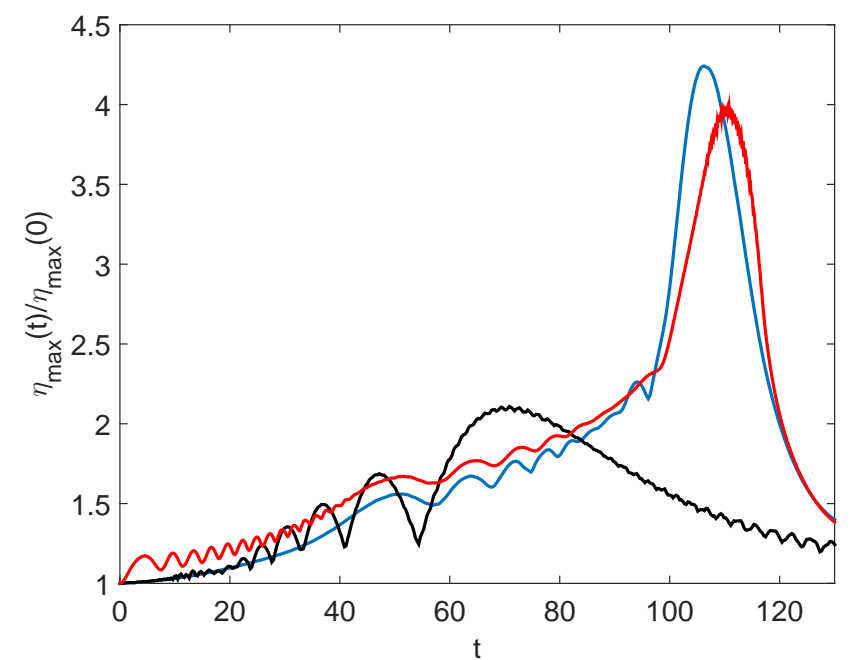

Fig. 4 (color online) Time evolution of the amplification factor with vorticity effect ( $\Omega=$ 0.5). Generalised Whitam equation (blue solid line), BIEM (red solid line) and KdV equation (black solid line) 


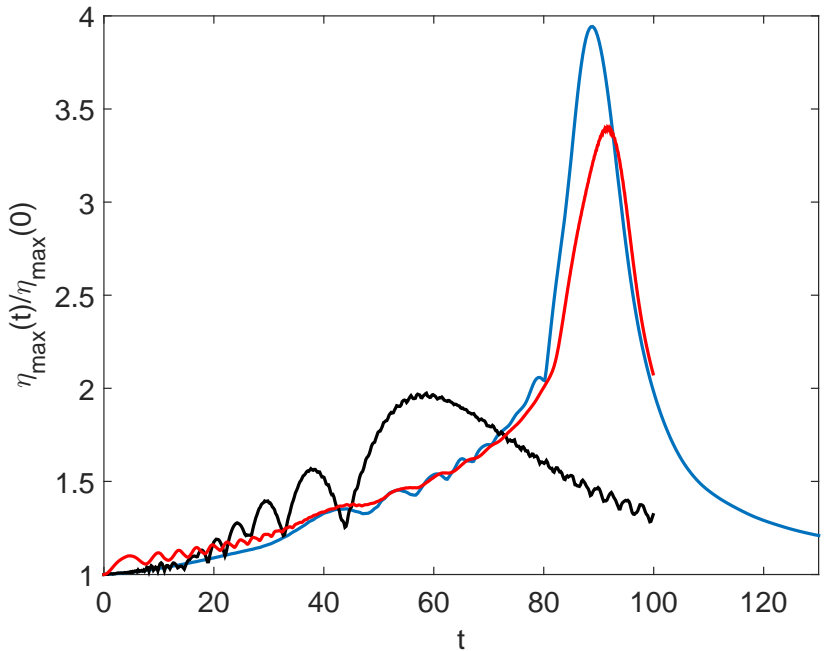

Fig. 5 (color online) Time evolution of the amplification factor with vorticity effect ( $\Omega=0.25$ ). Generalised Whitam equation (blue solid line), BIEM (red solid line) and $\mathrm{KdV}$ equation (black solid line)

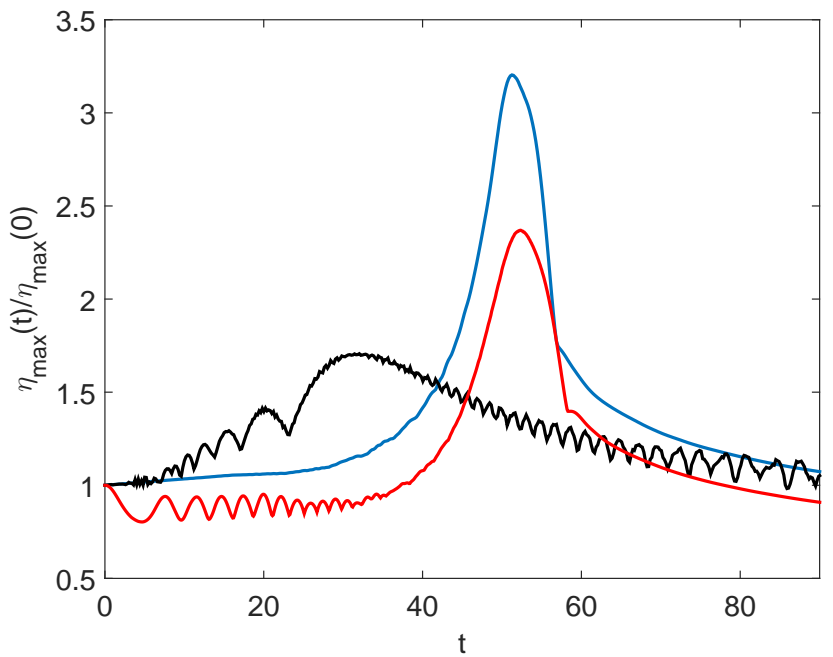

Fig. 6 (color online) Time evolution of the amplification factor with vorticity effect ( $\Omega=$ -0.5 ). Generalised Whitham equation (blue solid line), BIEM (red solid line) and KdV equation (black solid line) 


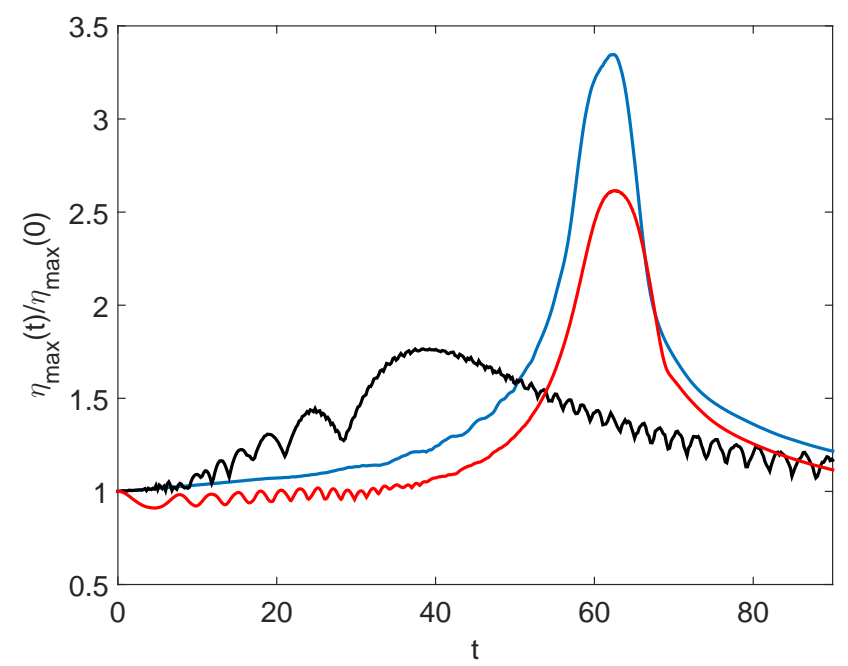

Fig. 7 (color online) Time evolution of the amplification factor with vorticity effect ( $\Omega=$ $-0.25)$. Generalised Whitam equation (blue solid line), BIEM (red solid line) and KdV equation (black solid line)

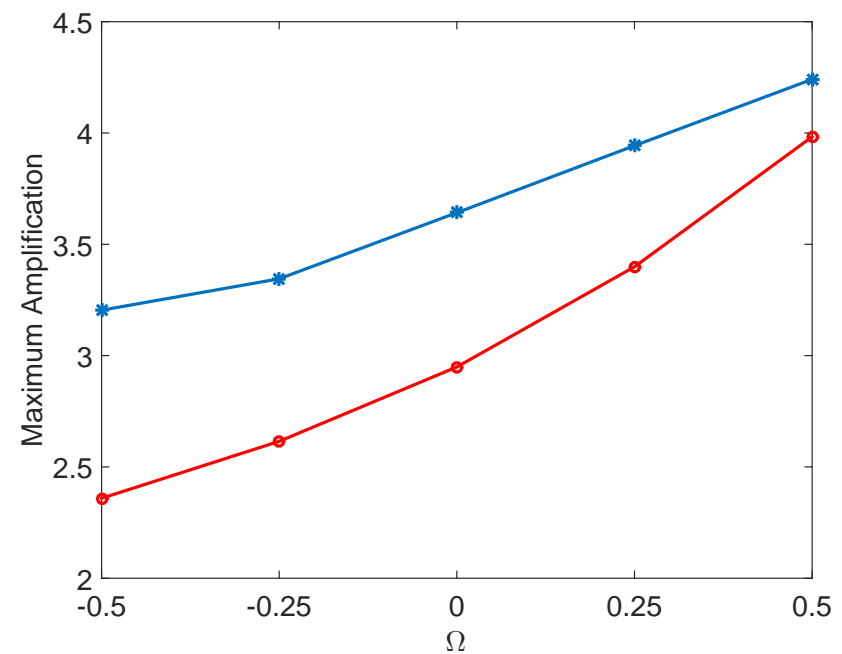

Fig. 8 (color online) Maximum amplification factor at the focusing time as a function of the shear intensity of the current. Generalised Whitam equation (blue solid line), BIEM (red solid line) 


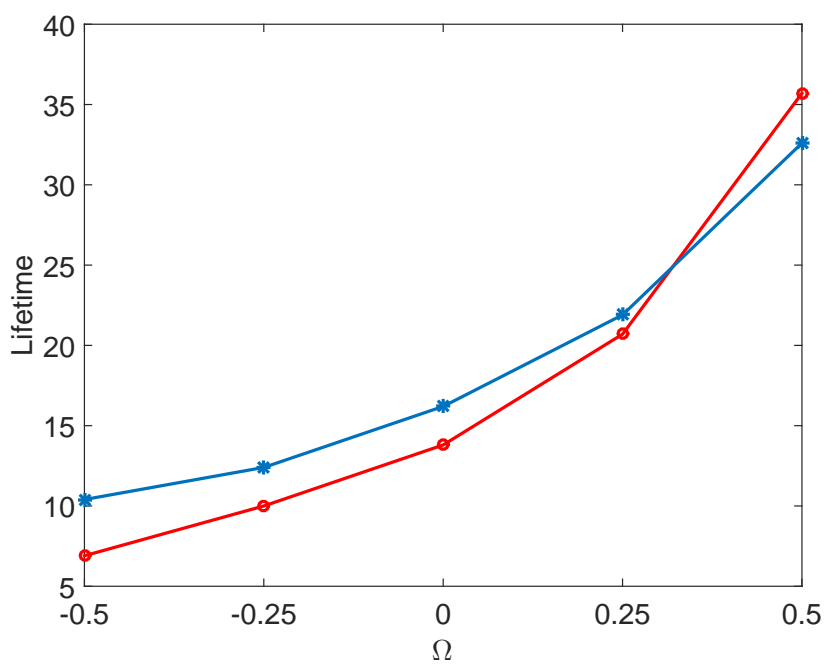

Fig. 9 (color online) Rogue wave duration as a function of the shear intensity of the current. Generalised Whitam equation (blue solid line), BIEM (red solid line)

\section{Conclusion}

The effect of an underlying vortical current on two-dimensional rogue wave properties has been investigated by using two different approaches in shallow water. One is based on a new approximate equation, the generalised Whitham equation with constant vorticity which is fully nonlinear and fully linear dispersive whereas the other, the BIEM with constant vorticity, is fully nonlinear and fully nonlinear dispersive. Besides the study on vorticity effect on rogue waves, it is shown that the results of the generalised Whitham equation with vorticity are in agreement with those of the BIEM demonstrating that this new single nonlinear equation is an efficient model for the investigation of nonlinear long waves on vertically sheared current of constant vorticity.

The numerical simulations carried out with all the approaches have shown that the presence of vorticity modifies the rogue wave properties significantly. The maximum of amplification factor of the surface elevation increases as the shear intensity of the current increases. The lifetime of extreme wave event follows the same tendency. 
Acknowledgements The authors would like to thank the French DGA, who supported this work through the ANR grant ANR-13-ASTR-0007.

\section{References}

Chambarel J, Kharif C, Kimmoun O (2010) Generation of two-dimensional steep water waves on finite depth with and without wind. Eur J Mech B/Fluids 29(2):132-142

Choi W (2003) Strongly nonlinear long gravity waves in uniform shear flows. Phys Rev E 68:026,305

Dysthe Kl, Krogstad HE, Muller P (2008) Oceanic rogue waves. Annu Rev Fluid Mech 40:287-310

Freeman N, Johnson R (1970) Shallow water waves on shear flows. J Fluid Mech 42 (2):401409

Hur VM, Johnson MA (2015) Modulational instability in the whitham equation with surface tension and vorticity. Nonlinear Analysis 129:104-118

Kharif C, Abid M (2017) Whitham approach for the study of nonlinear waves on a vertically sheared current in shallow water. J Fluid Mech (in revision)

Kharif C, Pelinovsky E (2003) Physical mechanisms of the rogue wave phenomenon. Eur J Mech B/Fluids 22:603-634

Kharif C, Pelinovsky E, Talipova T (2000) Formation de vagues géantes en eau peu profonde. CRAS 328 (IIb):801-807

Kharif C, Pelynovsky E, Slunyaev A (2009) Rogue waves in the ocean. Springer

Merkoune D, Touboul J, Abcha N, Mouazé D, Ezersky A (2013) Focusing wave group on a current of finite depth. Nat Hazards Earth Syst Sci 13:2941-2949

Nwogu OG (2009) Interaction of finite-amplitude waves with vertically sheared current fields. J Fluid Mech 627:179-213

Onorato M, Residori S, Bertolozzo U, Montina A, Arecchi F (2013) Rogue waves and their generating mechanisms in different physical contexts. Phys Rep 528:47-89

Pelinovsky E, Sergeeva A (2006) Numerical modeling of kdv random wave field. Eur J Mech B/Fluids 25:425-434

Pelinovsky E, Talipova T, Kharif C (2000) Nonlinear dispersive mechanism of the freak wave formation in shallow water. Physica D 147:83-94

Peterson P, Soomere T, Engelbrecht J, van Groesen E (2003) Interaction solitons as a possible model for extreme waves in shallow water. Nonlin Proc Geophys 10:503-510

Simmen JA (1984) Steady deep-water waves on a linear shear current. PhD thesis, California Institute of Technology, Pasadena, Californie

Simmen JA, Saffman PG (1985) Steady deep-water waves on a linear shear current. Stud Appl Math 73:35-57 
Soomere T, Engelbrecht (2005) Extreme evaluation and slopes of interacting solitons in shallow water. Wave Motion 41:179-192

Talipova T, Kharif C, Giovanangeli J (2008) Modelling of rogue wave shapes in shallow water. Springer, Eds. Pelinovsky E. and Kharif C.

Toffoli A, Onorato M, Osborne A, Monbaliu J (2006) Non-gaussian properties of surface elevation in crossing sea states in shallow water. Proc Int Conf Coast Eng (ICCE06 pp $782-790$

Touboul J, Kharif C (2010) Two-dimensional direct numerical simulations of the dynamics of rogue waves under wind action, Advances in numerical simulation of nonlinear water waves, vol 11, The world Scientific Publishing Co., London, chap 2

Touboul J, Kharif C (2016) Effect of vorticity on the generation of rogue waves due to dispersive focusing. Natural Hazards 84(2):585-598

Touboul J, Pelinovsky E, Kharif C (2007) Nonlinear focusing wave groups on current. Journ Korean Soc Coast And Ocean Engineers 19 (3):222-227

Whitham G (1974) Linear and nonlinear waves. John Wiley \& Sons 